\title{
Extracción de Características en Equipos Eléctricos basada en Medida de Vibraciones
}

\author{
Vicente Pastoriza, Francisco Poza, Perfecto Mariño y Fernando Machado \\ Univ. de Vigo, Dpto. Tecnología Electrónica, E.T.S. Ing. Industriales, Campus Universitario s/n, \\ 36310 Vigo-España (e-mail: \{vpastoriza, fpoza, pmarino, fmachado\}@uvigo.es)
}

\begin{abstract}
Resumen
Se desarrolló un sistema portátil de monitorización del estado de cambiadores de tomas en carga de transformadores de potencia en servicio, mediante la realización de inspecciones no invasivas. El sistema construido se basó en un instrumento virtual para medir señales de tensión, corriente y vibraciones durante los cambios de toma. El diagnóstico del estado de funcionamiento se realizó mediante análisis de vibraciones. El sistema portátil fue probado realizando inspecciones en los transformadores de varias subestaciones. De los resultados se concluye que el instrumento virtual facilita la realización de este tipo de inspecciones. Además, un estudio efectuado sobre un único cambiador de tomas en carga no descartó la posibilidad de estimar su estado de funcionamiento a partir de las vibraciones registradas con el sistema desarrollado. Sin embargo, para garantizar esto sería necesario efectuar una campaña de medidas más extensa.
\end{abstract}

Palabras clave: equipos eléctricos, análisis de vibraciones, cambiador de tomas en carga, transformador de potencia

\section{Feature Extraction from Electrical Equipment based on Vibration Measurements}

\begin{abstract}
A portable system for the condition monitoring of on-load tap changer in on-line power transformers by non-invasive inspections was developed. The system was based on a virtual instrument for measuring signals of voltage, current and vibration that a tap change produces. The the diagnosis of the functioning condition was done using vibration analysis. Inspections on transformers of several substations were done to test the proposed portable system. From the results, it is concluded that the virtual instrument facilitates this kind of inspection. Besides, a study carried out on a sole on-load tap changer did not discard the possibility of estimating the operating condition of on-load tap changers from the measured vibration signals. However, in order to guarantee this it will be necessary to performed more tests.
\end{abstract}

Keywords: electrical equipment, vibration analysis, on-load tap changers, power transformer 


\section{INTRODUCCIÓN}

Los cambiadores de tomas en carga de transformadores de potencia (CTC) son equipos electromecánicos encargados de regular la energía eléctrica proporcionada por los sistemas de distribución de electricidad a las redes de suministro eléctrico. Los CTCs controlan la tensión suministrada en función de la demanda de consumo. Esta regulación es efectuada por los CTCs mediante un conjunto de contactos eléctricos que permiten cambiar la relación de entrada/salida del transformador, un mecanismo de accionamiento a motor que mueve dichos contactos, y una unidad de control que determina la posición que deben tener los contactos en cada momento. Todo el equipo, a excepción de la unidad de control, trabaja inmerso en aceite dieléctrico.

EI CTC, debido a que es el único equipo mecánico con partes móviles de un transformador de potencia, es el componente más caro, con un mantenimiento más intensivo, y el que causa más averías (CIGRE, 1983; Kang et al., 2000). Se estima que la tercera parte de las averías en transformadores puede ser debida al fallo de su CTC (Dominelli et al., 2006). Las causas de avería mas frecuentes son el desgaste de los contactos eléctricos y problemas en el mecanismo de accionamiento a motor. Durante los cambios de toma se producen eventos eléctricos y mecánicos que van paulatinamente desgastando los contactos y degradando la resistencia dieléctrica del aceite: se producen descargas eléctricas que queman el aceite formando acumulaciones de carbón y los contactos desprenden partículas metálicas en el aceite debido a fricción mecánica. Esta progresiva contaminación del aceite con carbón y partículas metálicas va modificando las características dieléctricas del aceite, lo que favorece la aparición de descargas eléctricas cada vez más intensas, llegándose finalmente a la avería del transformador (Simas et al., 2005).

En la actualidad, el mantenimiento de un CTC se realiza mediante inspecciones periódicas después de un número fijo de cambios de toma (preventivo) o cuando se produce una avería (correctivo). Un inconveniente de este mantenimiento preventivo es que resulta caro y lento ya que para efectuar la inspección es necesario dejar el equipo fuera de servicio. Otro inconveniente adicional es que puede ocurrir que un equipo en buen estado, que podría haber estado más tiempo en funcionamiento, sea retirado del servicio para inspección o que un equipo alcance un estado defectuoso antes de dicha inspección, lo que podría provocar una avería. Por ello, resulta interesante desarrollar un mantenimiento del CTC basado en inspecciones que permitan diagnosticar su estado sin tener que ponerlo fuera de servicio. Estas inspecciones no invasivas suponen reducciones considerables en costes y tiempo, lo que a su vez hace viable la realización de inspecciones con más frecuencia. Esto último repercute en una disminución en el riesgo de averías ya que permite efectuar un seguimiento del progresivo desgaste que sufren los contactos del CTC con el tiempo y pronosticar su futuro fallo (mantenimiento predictivo).

En la literatura, uno de los métodos más populares de monitorización no invasiva del estado de un CTC en servicio es el basado en el análisis del gas disuelto en aceite (Wang et al., 2000). Otros métodos que han resultado efectivos están basados en el análisis de las vibraciones medidas durante el tiempo que dura un cambio de toma (Bengtsson et al., 1998; Foata et al. 2000; Kang et al., 2000; Kang y Birtwhistle 2001, 2003; Almeida et al., 2005; Simas et al., 2005; Wegelin et al., 2005). Actualmente, también se está investigando el uso de trazadores químicos para determinar el desgaste en los contactos del CTC (Dominelli et al., 2006).

El trabajo presentado en este artículo forma parte de un proyecto de ámbito industrial para desarrollar un sistema de monitorización de estado de CTCs de transformadores de potencia en servicio (Rivas et al., 2005). No se pretende un sistema físicamente ubicado en un único CTC para realizar su monitorización continua. Al contrario, uno de los requisitos del proyecto es que dicho sistema sea portátil, de tal forma que pueda ser usado en CTCs de transformadores de potencia situados en estaciones de transformación de energía eléctrica distribuidas por distintas zonas geográficas. Este proyecto, que se encuentra actualmente en marcha, consta de dos fases. La primera comprende el diseño y construcción de un sistema portátil de monitorización de estado de CTCs en servicio mediante la realización de inspecciones no invasivas usando un instrumento virtual. Además incluye un estudio preliminar sobre la realización del diagnóstico de estado de los CTCs inspeccionados a partir del análisis de vibraciones medidas con el instrumento virtual y utilizando 
para ello algunos de los métodos más conocidos y efectivos de la literatura. La segunda fase del proyecto consiste en la realización de una campaña de medidas extensa en distintos CTCs, su análisis y la obtención para cada modelo de CTC de unos patrones de referencia que permitan estimar su estado de funcionamiento. En esta fase, y en función de los resultados obtenidos en la primera fase, se contempla la posibilidad de probar nuevas técnicas de análisis de vibraciones.

Este artículo se centra exclusivamente en la primera fase del proyecto, que es la que ha finalizado. De los resultados obtenidos en las pruebas de campo realizadas en transformadores de varias subestaciones se concluye que el instrumento virtual portátil desarrollado facilita en gran medida la realización de este tipo de inspecciones no invasivas basadas en la medida de vibraciones. Además, se ha estudiado la viabilidad de realizar una diagnosis del estado de un CTC a partir de las vibraciones registradas con el instrumento virtual. En concreto, se ha contrastado la coherencia de diagnóstico aplicando tres métodos: Kang y Birtwhistle (2001, 2003), Almeida et al. (2005), y Wegelin et al. (2005). De dicho estudio se concluye que no se puede descartar dicha posibilidad, aunque para garantizarla es necesaria una campaña de medidas más extensa.

\section{METODOLOGÍA PROPUESTA}

Como se ha explicado en la introducción, se pretende realizar el diagnóstico de estado de un CTC en servicio mediante inspecciones no invasivas. En este trabajo se define una inspección como un conjunto de ensayos, donde un ensayo consiste en el registro de señales de tensión, corriente, y vibración durante un cambio entre dos tomas de carga adyacentes. Una inspección es determinada especificando la toma inicial, la toma final y el número de veces que se desea repetir esa secuencia. La inspección, así configurada, contiene todos los ensayos necesarios para ir de la toma inicial a la final, y viceversa, el número de veces previamente asignado. Además debido a la estructura del CTC, se pueden realizar ensayos de cambio de la toma actual a la adyacente superior (sentido ascendente) o a la adyacente inferior (sentido descendente).

A continuación, se describe la estructura física y la programación del instrumento virtual desarrollado para efectuar las inspecciones (para una mayor información consultar Poza et al., 2006). Asimismo, se muestran los detalles fundamentales de los tres técnicas propuestas (Introducción) para realizar la diagnosis del estado de funcionamiento.

\section{Adquisición de datos}

La parte de adquisición de datos del sistema se encarga de realizar la medida de diferentes variables relativas al funcionamiento del CTC. El diseño físico empleado para la adquisición de datos incluye un conjunto de sensores para la medida del fenómeno físico, equipos de acondicionamiento y una tarjeta de adquisición de datos. El sistema utiliza 8 sensores analógicos: 4 sensores de efecto Hall para medir las tensiones y corrientes en dos de las fases del motor de accionamiento del CTC, y 4 acelerómetros piezoeléctricos situados sobre la carcasa del transformador en zonas próximas al CTC para medir las vibraciones que se producen durante un cambio de toma. La tarjeta de adquisición de datos empleada permite muestrear las señales de cada uno de los 8 sensores a una frecuencia de $50 \mathrm{KS} / \mathrm{s}$, lo que hace posible analizar hasta el armónico de $25 \mathrm{KHz}$ de las vibraciones medidas. Como la adquisición debe durar todo el tiempo que el motor del CTC permanece en operación, de 5 a 6 segundos, y como los datos suministrados por los controladores de la tarjeta son de doble precisión ( 8 bytes), resulta que el tamaño de los datos adquiridos por los 8 sensores durante un ensayo es del orden de $20 \mathrm{MB}$.

\section{Almacenamiento de datos}

Para poder obtener datos fiables de cada ensayo de cambio de toma, es necesario repetir varias veces cada uno de ellos. Esto provoca que los requisitos de almacenamiento de datos sean elevados. Así por ejemplo, para un CTC con 20 tomas (por tanto 19 cambios entre tomas), del que se quiera ensayar 5 veces cada cambio de toma tanto en sentido ascendente como descendente, se trabaja con datos del orden de 3,5GB. 
Los datos adquiridos se almacenan en una base de datos con tres tablas: una para almacenar las características de los diferentes transformadores, otra para almacenar las características de los distintos CTCs y otra para almacenar los datos de los ensayos. De esta forma, se consigue una gestión rápida y sencilla de los datos de los ensayos.

\section{Programación del instrumento virtual}

El instrumento virtual se ha desarrollado utilizando el entorno gráfico de programación LabVIEW 7.1 de National Instruments (NIC, 2003a, 2003b; Lázaro y del Río, 2005), y es el encargado de realizar la adquisición y almacenamiento de los datos. La interfaz de usuario del instrumento virtual está formada por varias pestañas (Fig. 1). A continuación se describe la forma de realizar una inspección.

En primer lugar se selecciona el transformador para inspección en la pestaña "Datos generales". Este transformador y su CTC asociado deben haber sido definidos previamente en la base de datos. Una vez seleccionado se muestran los datos del CTC correspondiente a ese transformador, así como los datos de los distintos ensayos realizados en este CTC. En esta pestaña también se configuran los datos necesarios para realizar una inspección: toma inicial, toma final y número de repeticiones. Además, en la pestaña "Adquisición", el instrumento virtual permite al usuario definir una adquisición de datos automática con distintas condiciones de inicio y fin de la adquisición. En la pestaña "Información ensayo" se indica al usuario que el sistema está esperando para iniciar la adquisición de los datos, en ella también se informa de la toma actual de partida, de la toma de llegada, y del número de ensayo que se va a efectuar. A continuación el usuario inicia el cambio de toma deseado accionando manualmente el pulsador apropiado en el exterior del armario de accionamiento y mando del CTC. Finalmente, el instrumento virtual crea un archivo con los datos adquiridos y almacena en la base de datos la información correspondiente al ensayo. Este proceso se repite hasta que se han realizado todos los ensayos de la inspección (todos los ensayos de cambio de toma desde la inicial a la final, y viceversa, el número de veces configurado).

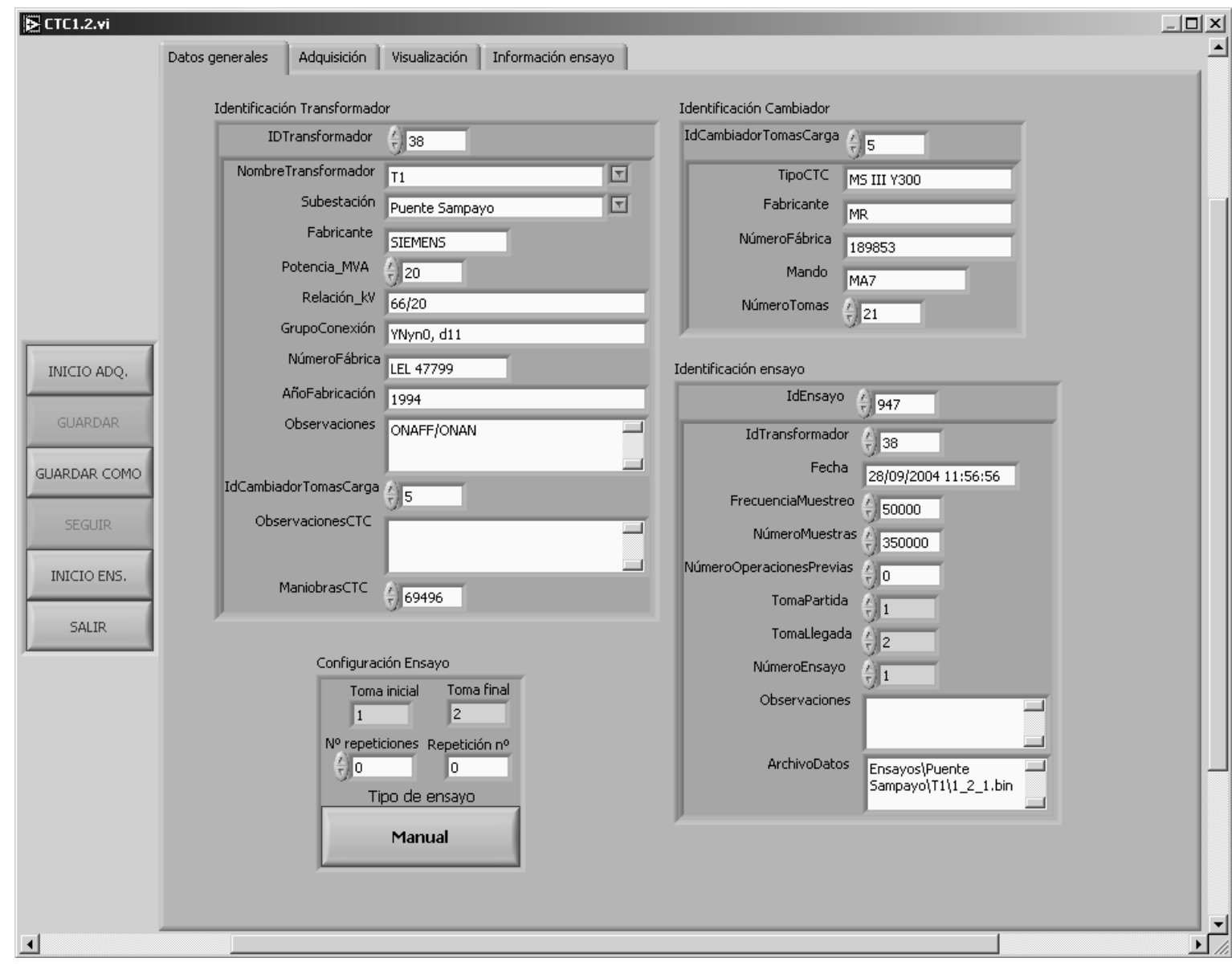

Fig. 1: Interfaz gráfica de usuario de la aplicación desarrollada (pestaña "Datos generales") 
Con esta interfaz, el instrumento virtual facilita en gran medida la realización de inspecciones, ya que el usuario solamente debe primero configurar el número de toma inicial y el de toma final, opcionalmente el número de veces que se ejecutará cada cambio de toma y después activar el pulsador de cambio de toma correspondiente.

\section{Estimación del estado de un CTC}

La operación de cambio de toma de carga de un CTC produce una serie bien definida de vibraciones. En consecuencia, varios autores (Introducción) han desarrollado métodos para diagnosticar el estado de un CTC a partir de las vibraciones medidas durante el cambio de toma de carga. En este trabajo se han aplicado tres de estos métodos:

El método de Kang y Birtwhistle $(2001,2003)$ se basa en la aplicación de una transformada de ondículas continua (TOC) (Mallat, 1999) para extraer unos indicadores del estado de funcionamiento del CTC a partir de la detección de las principales singularidades de las vibraciones medidas (método K03). Para ello, en primer lugar se aplica una transformada Hilbert a la señal de vibración y se obtene su envolvente. Después a esta envolvente normalizada se le aplica una TOC. Finalmente, se halla el conjunto de líneas de máximo vertical de la TOC del que se extraen las crestas dominantes, que se corresponden con las principales ráfagas de las vibraciones. La fuerza de estas crestas y el intervalo de tiempo entre ellas son utilizados como indicadores del estado del CTC. Se utiliza como TOC la ondícula Gaussiana compleja de primer orden en lugar de la Symlet de cuarto orden propuesta por los autores del método original (Misiti et al., 2007). Con esta modificación, se obtuvo una detección más robusta de las crestas dominantes en las vibraciones de los ensayos realizados.

Almeida et al. (2005), han desarrollado un sencillo método de monitorización no invasiva (método A05) fundamentado en el análisis de vibraciones usando una transformada de ondículas discreta (TOD). Primero se realiza una descomposición multinivel de la señal de las vibraciones en coeficientes de aproximación y detalle aplicando una TOD (Mallat, 1999). A continuación se define un coeficiente de energía para cada nivel de resolución, como la relación de energía entre la secuencia de coeficientes de aproximación y de detalle en ese nivel. El estado de funcionamiento es evaluado en función de la dependencia de los coeficientes de energía normalizados (CEN) con el nivel de resolución: por ejemplo, contactos desgastados muestran una tendencia de crecimiento de los CEN con el nivel, y viceversa para contactos nuevos.

Wegelin et al. (2005), han propuesto un procedimiento no invasivo mediante el análisis Prony de las vibraciones (método W05). El método Prony es una técnica paramétrica para ajustar una señal por sinusoides amortiguadas. Basándose en los parámetros obtenidos usando este método definen un índice de dispersión de energía (IDE) para estimar el estado de cada contacto de un CTC: así los contactos desgastados presentan mayor dispersión en el índice que los contactos nuevos. Además, establecen un valor de IDE umbral para determinar el estado del contacto del CTC: Ios contactos desgastados tienen valores de IDE por debajo del umbral, y viceversa para los contactos nuevos. Se ha aplicado el método con dos modificaciones respecto al original debido al hecho de utilizar una frecuencia de muestreo de $10 \mathrm{KHz}$ en lugar de los $44.1 \mathrm{KHz}$ del original. Dichas modificaciones fueron: utilizar en el cálculo de IDE una constante de dispersión $\sigma$ de 11338 en vez de 50000 del original, y un valor de IDE umbral de $3.48 \cdot 10^{-5}$ en vez de los $7.9 \cdot 10^{-6}$.

\section{RESULTADOS Y DISCUSIÓN}

Para validar el desempeño y robustez del instrumento virtual se realizaron inspecciones en CTCs de transformadores en varias subestaciones. Todas las pruebas de campo efectuadas pusieron de manifiesto que la utilización del instrumento virtual desarrollado facilitaba la realización de las inspecciones.

Además, también se hizo un estudio preliminar sobre la viabilidad de realizar una diagnosis del estado de los CTCs a partir de las vibraciones registradas con el instrumento virtual en uno de los CTCs inspeccionados. Este estudio presentó el inconveniente de no poder conocer con certeza el estado real de ninguno de los CTCs ya que no fue posible hacer coincidir sus inspecciones con 
períodos de mantenimiento, debido a que la frecuencia de mantenimiento es de varios años. Esta limitación fue solventada efectuando una estimación del estado por tres métodos distintos: K03, A05, y W05; y contrastando la coherencia de los resultados obtenidos por dichos métodos. La elección del CTC sometido a estudio fue hecha al azar.

En la Fig. 2 se visualizan las señales resultantes del procesado y análisis de la señal de las vibraciones registrada en un ensayo de cambio de la toma 8 a la 9 del CTC aplicando el método K03. La gráfica superior muestra la vibración medida, la gráfica central muestra la envolvente de dicha vibración calculada usando la transformada Hilbert, y la gráfica inferior muestra las 4 crestas dominantes de la TOC aplicada a la envolvente. Aplicando esta técnica a vibraciones recogidas en ensayos en todos los cambios de toma del CTC se extrajeron unos indicadores de estado (fuerza de crestas e intervalo de tiempo entre crestas) bastante similares (Tabla 1). Sin embargo, dichos indicadores no se parecieron en nada a los obtenidos en Kang y Birtwhistle (2001, 2003), ni para CTCs con contactos nuevos ni con contactos desgastados, debido a que las firmas de las señales de vibración registradas en nuestros ensayos fueron totalmente diferentes a las suyas. Por ello no fue posible hacer un diagnóstico del estado del CTC con este método.

En cambio, como las firmas de las señales de vibración utilizadas por Almeida et al. (2005) y Wegelin et al. (2005), eran similares a las registradas en nuestros ensayos, consideramos acertado aplicar a nuestro caso de estudio los criterios de diagnóstico de estado de ambos métodos con las modificaciones descritas en la sección anterior. En la Fig. 3 se muestra el resultado obtenido con el método A05, apreciándose claramente para todos los contactos que sus CEN crecen con el nivel de resolución y por tanto implicando que los contactos del CTC están desgastados. Análogamente, la Fig. 4 presenta el IDE calculado para cada contacto del CTC con el método W05. Tanto el grado de

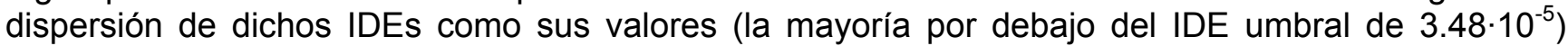
indicaron que el CTC tenía la mayoría de los contactos desgastados.

La coincidencia en el diagnóstico de estado del CTC aportado por estos dos métodos da fiabilidad a la estimación obtenida. Además, este resultado fue reforzado por el elevado número de operaciones de cambio de toma que llevaba el CTC en el momento de la inspección lo que justificaría la existencia de contactos desgastados por el uso. Finalmente, debe destacarse que si consideramos válida esta estimación entonces los indicadores de estado extraídos con el método K03 podrían ser un patrón de referencia de contactos desgastados, aunque haría falta aplicar esta técnica sobre CTCs con contactos nuevos y comprobar que los indicadores obtenidos presentan un patrón distinto.
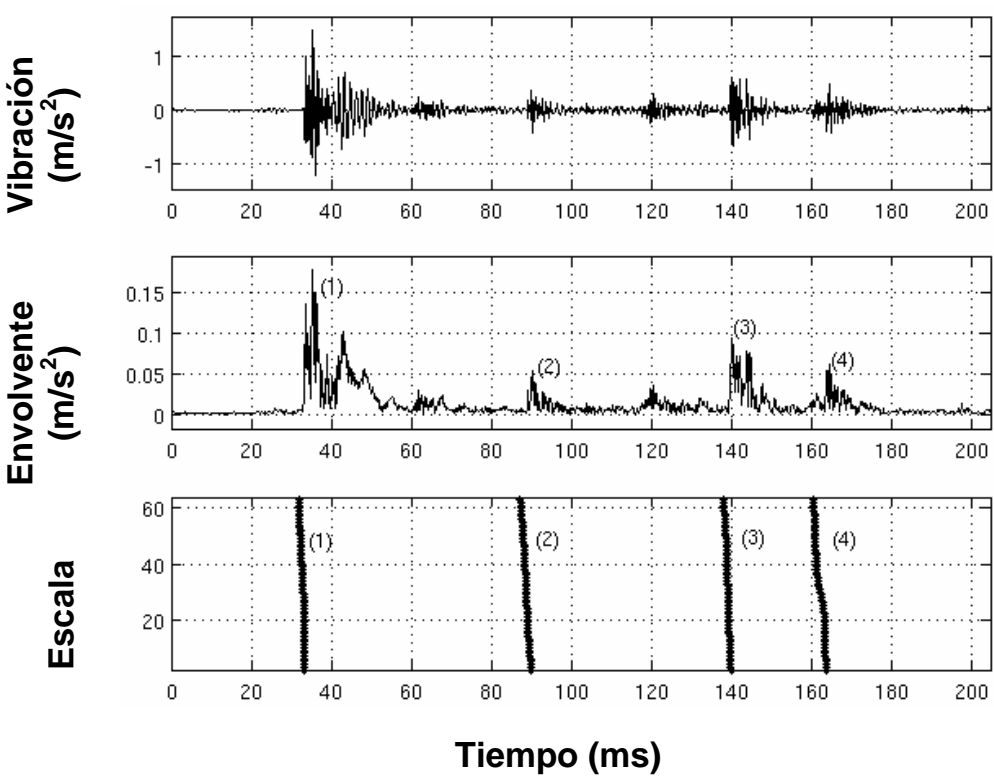

Fig. 2: Ejemplo de análisis de la vibración medida en un ensayo de cambio de la toma de carga 8 a la 9 del CTC: vibración medida (arriba); envolvente de la vibración medida (centro); cuatro crestas dominantes extraídas de la envolvente (abajo)
Tabla1: Variabilidad de los indicadores de estado de funcionamiento del CTC extraídos con el método $\mathrm{KO3}$ a partir de ensayos realizados sobre todos los cambios de toma del CTC ( $\bar{x}$, media; $\sigma$, desviación típica)

\begin{tabular}{|c|c|c|c|c|}
\hline \multirow{2}{*}{ cresta } & \multicolumn{2}{|c|}{ fuerza } & \multirow{2}{*}{\multicolumn{2}{|c|}{$\begin{array}{l}\text { tiempo entre } \\
\text { crestas (ms) }\end{array}$}} \\
\hline & $\overline{\bar{x}}$ & $\sigma$ & & \\
\hline \multirow{2}{*}{1} & \multirow{2}{*}{145.36} & \multirow{2}{*}{13.30} & $\bar{x}$ & $\sigma$ \\
\hline & & & 55.42 & 1.48 \\
\hline 2 & 22.11 & 7.85 & \multirow{2}{*}{50.92} & \multirow{2}{*}{0.48} \\
\hline \multirow{2}{*}{3} & \multirow{2}{*}{88.52} & \multirow{2}{*}{11.26} & & \\
\hline & & & \multirow[t]{2}{*}{22.05} & \multirow[t]{2}{*}{2.20} \\
\hline 4 & 31.17 & 16.19 & & \\
\hline
\end{tabular}




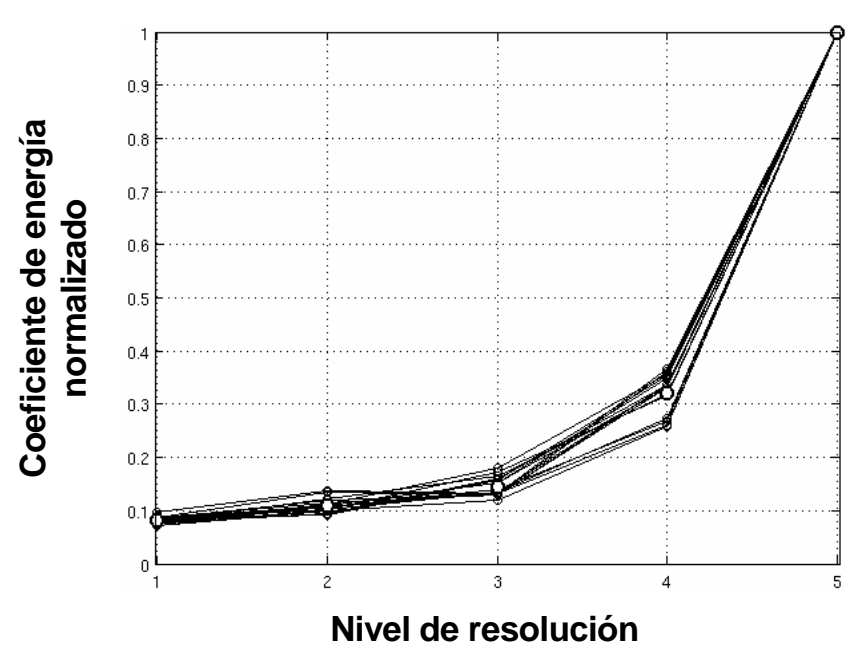

Fig. 3: Coeficientes de energía normalizados (CEN) en función del nivel de resolución para cada contacto de CTC (tendencia creciente para contactos desgastados)

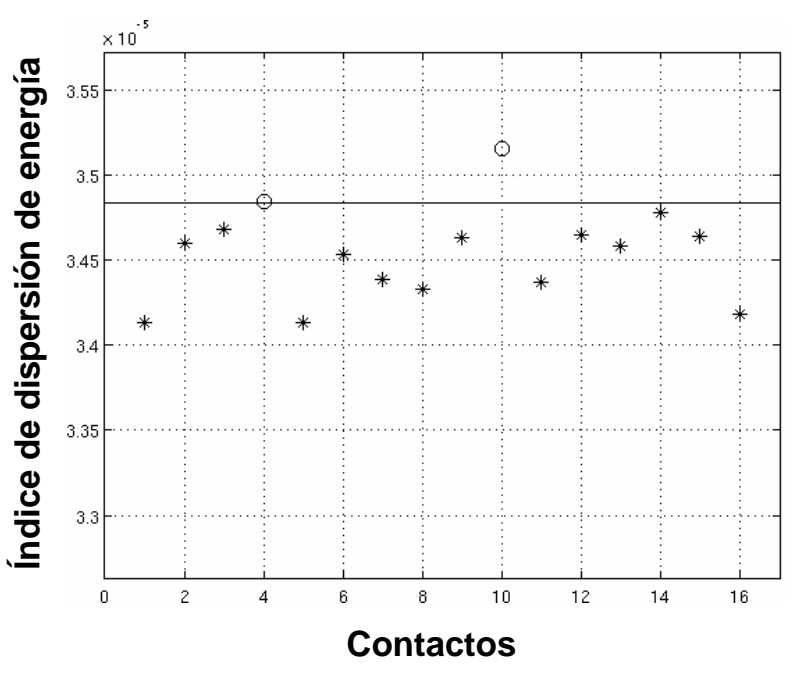

Fig. 4: Índice de dispersión de energía (IDE) para cada contacto del CTC ( $*$ contactos desgastados)

Por tanto, este estudio preliminar sobre un único CTC indica que no se puede descartar la viabilidad de estimar el estado de un CTC a partir de las vibraciones registradas con el sistema desarrollado. Para garantizar dicha viabilidad es necesario efectuar una campaña de medidas extensa en distintos CTCs de diferentes transformadores en distintas condiciones de funcionamiento, y donde se conozca con certeza su estado real. Como se ha comentado en la Introducción, todo esto constituye la segunda parte del proyecto.

\section{CONCLUSIONES}

De los resultados obtenidos, de su análisis y de su discusión, se pueden obtener las siguientes conclusiones sobre el sistema portátil de monitorización de estado de CTCs de transformadores de potencia en servicio desarrollado: 1) el diagnóstico de estado radica en el análisis de vibraciones medidas durante los cambios de toma; 2) el instrumento virtual diseñado facilita en gran medida la realización de inspecciones no invasivas basadas en la medida de vibraciones; 3 ) en base al estudio efectuado sobre un único CTC con los métodos de Kang y Birtwhistle (2001, 2003), Almeida et al. (2005), y Wegelin et al. (2005), no se puede descartar la posibilidad de estimar el estado de un CTC a partir de las vibraciones registradas por el sistema; 4) para garantizar dicha viabilidad es necesario efectuar una campaña de medidas más extensa.

\section{AGRADECIMIENTOS}

Este artículo se inscribe dentro de la línea de investigación del proyecto DPI2006-15625-C03-03 del Plan Nacional de I+D+i de 2006.

\section{REFERENCIAS}

Almeida, L.A.L., M. Fontana, F.A. Wegelin y L. Ferreira; A New Approach for Condition Assessment of On-Load Tap-Changers Using Discrete Wavelet Transform, Instrumentation and Measurement Technology Conference, IMTC 2005, 653-656, Ottawa, Canada, del 17 al 19 de Mayo (2005).

Bengtsson T., H. Kols, M. Foata y F. Léonard; Monitoring Tap Changer Operations, Cigre 1998, paper 12-209 (1998).

CIGRE SC 12 WG 12.05; An International Survey on Failures in Large Power Transformers in Service, ELECTRA: 88, 21-47 (1983). 
Dominelli, N., A. Rao y P. Kundur; Life Extension and Condition Assessment. Techniques for an Aging Utility Infrastructure, IEEE Power and Delivery Magazine: 4(3), 24-35 (2006).

Foata, M., R. Beauchemin y C. Rajotte; On-line Testing of On-Load Tap Changers with a Portable Acoustic System, 2000 IEEE ESMO - IEEE 9th International Conference on Transmission and Distribution Construction, Operation and Live-Line Maintenance Proceedings, 293-298, Montreal, Canada, del 8 al 12 de Octubre (2000).

Kang ,P., D. Birtwhistle, J. Daly y D. McCulloch; Non-invasive on-line condition monitoring of on-load tap-changers, Proceedings of IEEE Power Engineering Society Winter Meeting, 2223-2228, Singapore, Singapore, del 23 al 27 de Enero (2000).

Kang ,P. y D. Birtwhistle; Condition Assessment of Power Transformer On-Load Tap-Changers Using Wavelet Analysis, IEEE Transactions on Power Delivery: 16(3), 394-400 (2001).

Kang ,P. y D. Birtwhistle; Condition Assessment of Power Transformer On-Load Tap-Changers Using Wavelet Analysis and Self-Organizing Map: Field Evaluation, IEEE Transactions on Power Delivery: 18(1), 78-84 (2003).

Lázaro, A.M. y J. del Río; LabVIEW 7.1. Programación Gráfica para el Control de Instrumentación, 1326, Thomson, Madrid, España (2005).

Mallat, S.; A Wavelet Tour of Signal Processing, $2^{\text {a }}$ edición, 1-634, Academic Press, San Diego, USA (1999).

Misiti, M., Y. Misiti, G. Oppenheim y J.M. Poggi; Wavelet Toolbox 4 User's Guide (en línea), 2007. http://www.mathworks.com. Acceso: 8 de Diciembre (2007).

NIC; National Instruments Corporation, LabVIEW 7 Express. Measurements Manual, April 2003 ed., part Number: 322661B-01 (2003a).

NIC; National Instruments Corporation, LabVIEW 7 Express. User Manual, April 2003 ed., part Number: 320999E-01 (2003b).

Poza, F., P. Mariño, S. Otero y V. Pastoriza; OLTC Measurement in Electrical Grids, 49th International Midwest Symposium on Circuits and Systems, MWSCAS'06, 631-635, San Juan, Puerto Rico, del 6 al 9 de Agosto (2006).

Rivas, E., D. Urquiza y J. Burgos; Diagnóstico de Averías en Cambiadores de Tomas en Carga, VII Congreso Latinoamericano y IV Iberoamericano en Alta Tensión y Aislamiento Eléctrico, ALTAE'05, Panamá, Panamá, 26 al 30 de Julio (2005).

Simas, E.F., L.A.L. Almeida y A.C. Lima; Vibration Monitoring of On-Load Changers Using a Genetic Algorithm, Instrumentation and Measurement Technology Conference, IMTC 2005, 2288-2293, Ottawa, Canada, del 17 al 19 de Mayo (2005).

Wang, H., Y. Liu y P. Griffin; Artificial Intelligence in OLTC Fault Diagnosis Using Dissolved Gas-In Oil Information, IEEE, Power Engineering Society Summer Meeting: 4, 2422-2427, (2000).

Wegelin, F.A., R.S. Magalhães, L.A.L. Almeida y M. Fontana; Condition Monitoring of Power Transformers Using Acoustic Signal Prony's Analysis, Instrumentation and Measurement Technology Conference, IMTC 2005, 1384-1387, Ottawa, Canada, del 17 al 19 de Mayo (2005). 